\title{
Expression profiles uncover the relevance between colony stimulating factor-mediated signaling pathways in liver cells and partial hepatectomy-induced hepatic regeneration
}

\author{
X.G. Chen ${ }^{1}$ and C.S. $\mathrm{Xu}^{2,3}$ \\ ${ }^{1}$ Animal Science and Technology School, \\ Henan University of Science and Technology, Luoyang, China \\ ${ }^{2}$ College of Life Science, Henan Normal University, Xinxiang, China \\ ${ }^{3}$ Key Laboratory for Cell Differentiation Regulation, Xinxiang, China \\ Corresponding author: C.S. Xu \\ E-mail: cellkeylab@126.com
}

Genet. Mol. Res. 13 (3): 6356-6366 (2014)

Received June 24, 2013

Accepted December 13, 2013

Published February 14, 2014

DOI http://dx.doi.org/10.4238/2014.February.14.17

\begin{abstract}
Colony stimulating factors (CSF) have been considered to modulate liver regeneration (LR) after partial hepatectomy $(\mathrm{PH})$ at the tissue level. However, it remains unclear about precise mechanism of action of CSF in regeneration at the cellular level. Therefore, eight rat liver cell types were isolated by Percoll gradient centrifugation and magnetic beads. CSF-mediated signaling pathway genes were obtained by searching the related pathway databases and their expression profiles in 8 hepatic cell types were measured using rat Genome 230 2.0 Microarray. RT-PCR was performed to assess the reliability of chip results. The result showed a large difference in expression profiles of CSF-mediated signaling pathway genes between different cell types; most genes involved in CSF-mediated signaling pathways were mainly unregulated across liver cell samples. The implication of these genes in LR was analyzed by the bioinformatics and systems biology method.
\end{abstract}


According to chip results and gene synergy, a significant enhancement of the CSF3-mediated Pi3k/Akt pathway at 30-36 h in hepatocytes and at $24 \mathrm{~h}$ in biliary epithelial cells post-PH could be associated with active proliferation in these two cell types; the striking decrease in $\mathrm{Jak} / \mathrm{Stat}$ cascade activity in hepatic stellate cells at 2 and $12 \mathrm{~h}$ post$\mathrm{PH}$ or even inactive in dendritric cells during the whole LR implied that proliferation of these two cell types is possibly regulated by other signaling pathways. These data suggest the potential relevance of CSF in liver regeneration at the cellular level.

Key words: Liver regeneration; Rat Genome 230 2.0 Microarray; Partial hepatectomy; Colony stimulating factors

\section{INTRODUCTION}

Liver cells, normally in a quiescent state, have a unique capability to reenter the cell cycle in response to cell loss caused by surgical hepatectomy, chemical stimuli or ischemic damage (Christ and Pelz, 2013). Liver restoration, being critical for liver mass recovery, is a stringently regulated process, which is mainly segmented into two phases including initiation phase [2-6 h after partial hepatectomy $(\mathrm{PH})]$ and proliferation phase (6-72 $\mathrm{h}$ after $\mathrm{PH}$ ) according to cellular physiological activities (Ogiso et al., 2007; Chen et al., 2010a). A growing body of evidence suggests that, besides a major contribution of existing hepatocyte replication, liver repopulation also relies on hepatic oval cells and extrahepatic bone marrow-derived cells, which are activated and differentiate into various cells including hepatocyte (HC) and biliary epithelial cell (BEC) (Van Sweringen et al., 2011).

Recently, some researchers have indicated that the above biological processes are orchestrated by various cytokines, among which colony stimulating factors (CSF) are considered to play an important role in directly enhancing oval cell activation and promoting migration of extrahepatic progenitors into the liver (Piscaglia et al., 2007). CSF is defined as a group of pleiotropic glycoproteins, mainly composed of macrophage-CSF (M-CSF or CSF1), granulocyte-macrophage-CSF (GM-CSF or CSF2), and granulocyte-CSF (G-CSF or CSF3) (Kaushansky, 2006). This kind of cytokine not only can induce cell replication and differentiation, but also maintain cell survival (Akagawa et al., 2006). All actions of CSFs are mediated by the activation of intracellular signaling pathways when binding to their receptors. To the best of our knowledge, the binding of CSF1-3 to the corresponding receptors could stimulate phosphatidylinositol 3-kinase (Pi3k), thereby triggering the Pi3k/Akt cascade to control many physiological processes such as cell survival, differentiation and anti-apoptosis, or directly induce the caspase/cdc42 pathway to modulate the dynamic balance between opposing prosurvival and pro-apoptotic forces (Ruse and Knaus, 2006). In addition, they also mediate the $\mathrm{Jak} / \mathrm{Stat}$ and Ras/Raf/Mapk pathways, by which they play a role in cell growth and apoptosis inhibition (Bhanu et al., 2004) (Figure 1).

To date, some progress has been made in studying the role of CSF in liver regeneration (LR). For instance, Ishikawa et al. (2006) showed the beneficial action of M-CSF on tissue regeneration. Others have found that M-CSF and GM-CSF can directly regulate the differentiation of hematopoietic progenitor toward Kupffer cells, which can produce various 
growth factors and immunomodulators with stimulatory or inhibitory effects on LR after PH (Lin et al., 2008; Amemiya et al., 2011). Sidler et al. (2008) found that G-CSF administration promoted LR in an experimental liver remnant mouse model. However, the controversial observations from Ono et al. (2004) and Ogiso et al. (2007) suggested that G-CSF treatment caused impaired liver regeneration in mouse. Recently, a study by Meng et al. (2012) suggested that the altered expression of GM-CSF after PH can contribute to biliary remodeling after transplantation or partial hepatectomy by functional deregulation of the activity of key signaling intermediates involved in cell expansion and differentiation. Piscaglia et al. (2007) showed that G-CSF facilitates $2 \mathrm{AAF} / \mathrm{PH}$-induced hepatic regeneration in rats by promoting migration and proliferation of endogenous oval cells. In a study conducted by Kang et al. (2008), G-GSF showed an inhibitory effect on hepatocyte apoptosis by probably upregulating $\mathrm{Bcl} 2$ and reducing caspase 3 expression. Gaia et al. (2006) showed that G-CSF contributes to rat liver repair in the mechanism by increasing bone marrow-derived liver repopulation, or by activating endogenous oval cells. Anyway, all of the above findings came from tissue-level studies. Today, there are still no reports on their mechanism of action at the liver cell levels, and few reports on the CSF signaling pathway in different liver cell types. Therefore, in the present study, we aimed to assess the role of CSF during PH-mediated LR at the cellular level by determining the expression profiles of genes involved in the CSF-mediated signaling pathway in eight liver cell types after PH in rats using Rat Genome 230 2.0 Microarray.

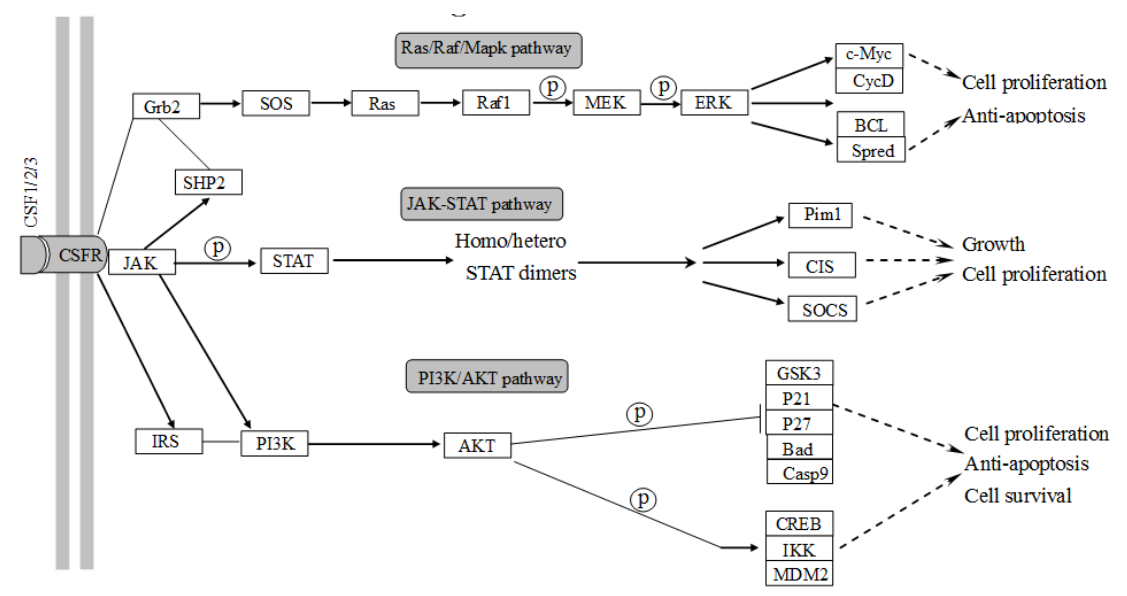

Figure 1. Schematic representation of CSF signaling pathway, in which three major pathways are involved. CSF binding activates CSFR and causes receptor phosphorylation on tyrosines. The phosphotyrosines recruit cytoplasmic signaling molecules. Among them, CSFR engages three parallel pathways, Jak/Stat, Ras/Raf/Mapk, and Pi3k/Akt.

\section{MATERIAL AND METHODS}

\section{Establishment of rat 2/3 hepatectomy model}

Sprague-Dawley rats were raised at the Animal Center of Henan Normal University in compliance with the current Laws of Animal Protection of China. A total of 90 adult Sprague- 
Dawley rats, weighing $190 \pm 20 \mathrm{~g}$, were selected and randomly divided into 15 groups with 6 rats in each group: 7 partially hepatectomized groups, 7 sham operation (SO) groups and 1 control group. Rats in $\mathrm{PH}$ groups underwent operation for 2/3 $\mathrm{PH}$ as previously described (Higgins and Anderson, 1931). Briefly, the left and median lateral liver lobes were surgically removed, and the rats after $\mathrm{PH}$ were sacrificed at 0, 2, 6, 12, 24, 30, 36, and $72 \mathrm{~h}$. Rats in SO groups underwent the operation process without removal of the liver lobes.

\section{Isolation and identification of eight kinds of liver cells}

Taking the partially hepatectomized rats $2 \mathrm{~h}$ postoperatively for example, the rats were ether anesthetized and sprayed with $75 \%$ alcohol for abdominal skin disinfection. The abdominal cavity was then opened to expose the liver, and rats were subjected to ligation of the inferior vena cava both below and above the liver, followed by catheterization of the liver portal vein. The dispersion of liver cells, and the separation and isolation of different liver cell types were performed according to the method previously described by Chen and $\mathrm{Xu}(2012)$.

\section{Microarray analyses}

Eight kinds of liver cells from regenerating rat liver at different recovery time after $\mathrm{PH}$ were used in cDNA microarray assays with the Affymetrix Rat Genome 2302.0 Microarray. Three experiments were performed on a single-total RNA preparation from one cell sample. Signal values are reported as mean values of three replicate experiments. cDNA microarray assays and statistical analyses of gene expression results were performed as described previously (Nault et al., 2013).

\section{Quantitative real-time PCR}

To validate the results obtained in the microarray experiments, qRT-PCR was performed. RNA samples for real-time PCR were prepared from regenerating liver at seven different recovery times. The primer sequence was designed by the Primer express 2.0 software according to mRNA sequences of the selected genes g6pc, jun, trim24, and myc, and then synthesized by Shanghai GeneCore BioTechnologies Co., and reverse-transcription of total RNA, first-strand cDNA synthesis of four genes and the generation of standard curves were done as previously described (Wang and $\mathrm{Xu}, 2010$ ).

\section{Gene synergy analysis}

A multivariate statistical-supporting mathematical model $\left(\mathrm{E}_{\mathrm{t}}\right)$ described by Wang et al. (2009) was used for assessing the timing, occurrence and strength of some biological activity governed by a group of functional-associated genes. Briefly, biological activity is strong when $\mathrm{E}_{\mathrm{t}}>\mathrm{E}_{0}$ (control) and shows a significant or extremely significant difference compared with $\mathrm{E}_{\mathrm{SO}}$ (sham operation); the activity is weak when $\mathrm{E}_{\mathrm{t}}<\mathrm{E}_{0}$ and shows a significant or extremely significant difference compared with $\mathrm{E}_{\mathrm{SO}}$; or activity is unchanged when $\mathrm{E}_{\mathrm{t}}=\mathrm{E}_{0}$ and shows no significant difference compared with $\mathrm{E}_{\mathrm{SO}}$. 


\section{RESULTS}

\section{qRT-PCR results}

In this study, the microarray expression data were validated by quantitative real-time PCR. Four genes including g6pc, jun, trim 24 , and myc were selected and analyzed for their expression changes by qRT-PCR. In all cases tested, the changes observed in the arrays were confirmed (Figure 2). The pattern of expression was very similar in both analyses, and the associated fold-change correlated closely.
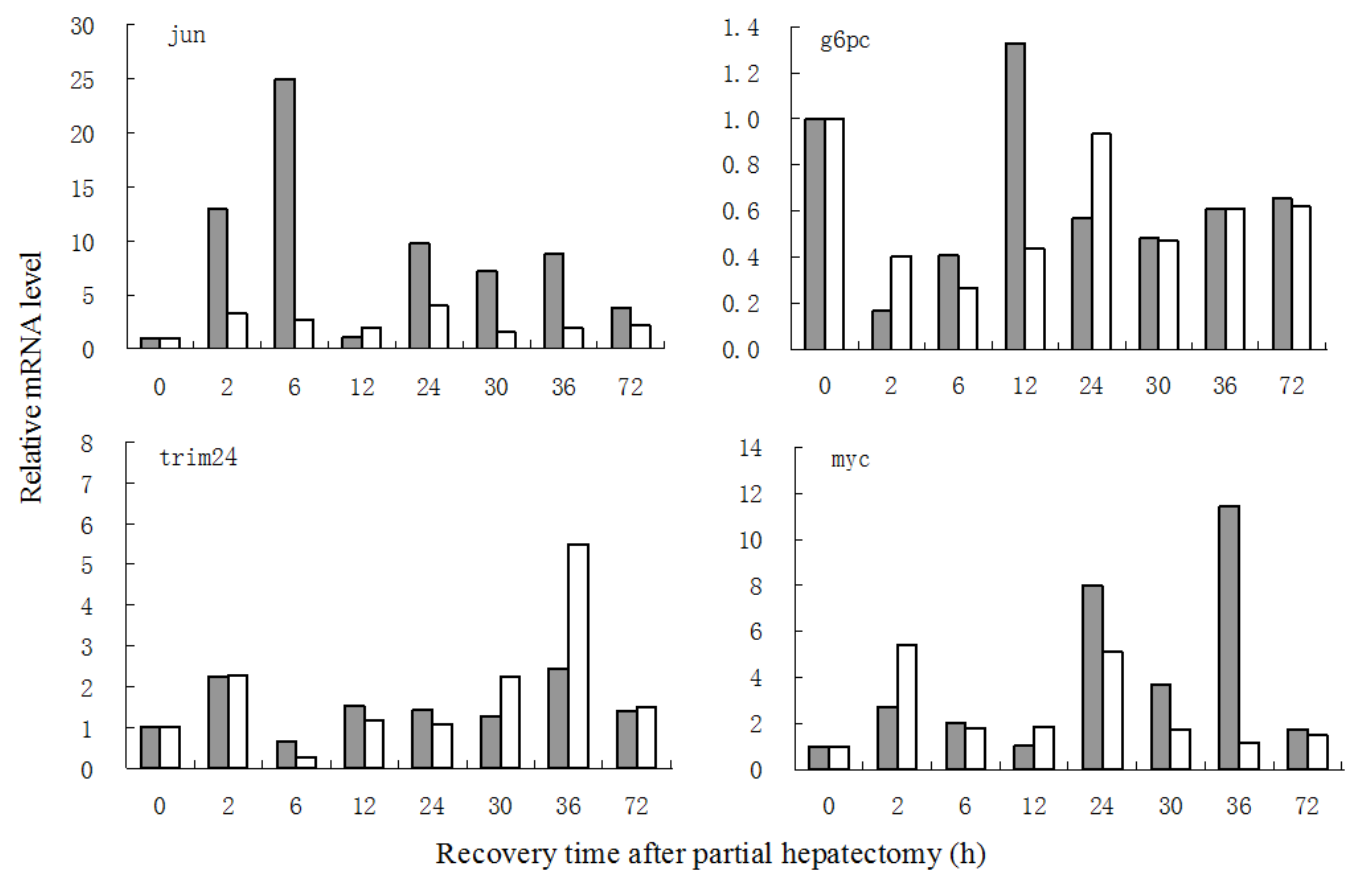

Figure 2. qRT-PCR analysis of mRNAs of four genes including g6pc, jun, trim 24 , and $m y c$. The relative mRNA levels at each time after partial hepatectomy in RT-PCR analysis (black bars) and microarray analysis (white bars) are shown for the mRNA of $g 6 p c$, jun, trim 24 , and $m y c$.

\section{Identification and expression profiles of CSF-mediated signaling pathway genes present on microarray}

To investigate how the expression of CSF-mediated signaling pathway genes responds to regeneration progression, we collected RNA samples from 8 different rat liver cell types at 7 different recovery times $(2,6,12,24,30,36$, and $72 \mathrm{~h})$ following PH in rats, and then used Rat Genome 2302.0 Microarrays to examine the temporal gene expression profile of these cell types. To rule out the possibility that any gene expression changes occur independently of liver regeneration progression, we obtained a sham-operation sample at corresponding recovery 
times in this study (Figure 3A). The independently triplicate hybridizations were performed for each sample using the microarray. Genes showing the similar expression trends at the same time in three chip analyses, having at least 2-fold or greater changes in expression between the experimental groups and the control group, and significant $(\mathrm{P} \leq 0.05$, using F-test) or very significant $(\mathrm{P} \leq 0.01)$ difference between $\mathrm{PH}$ and $\mathrm{SO}$ groups, were described as differentially expressed genes during LR.

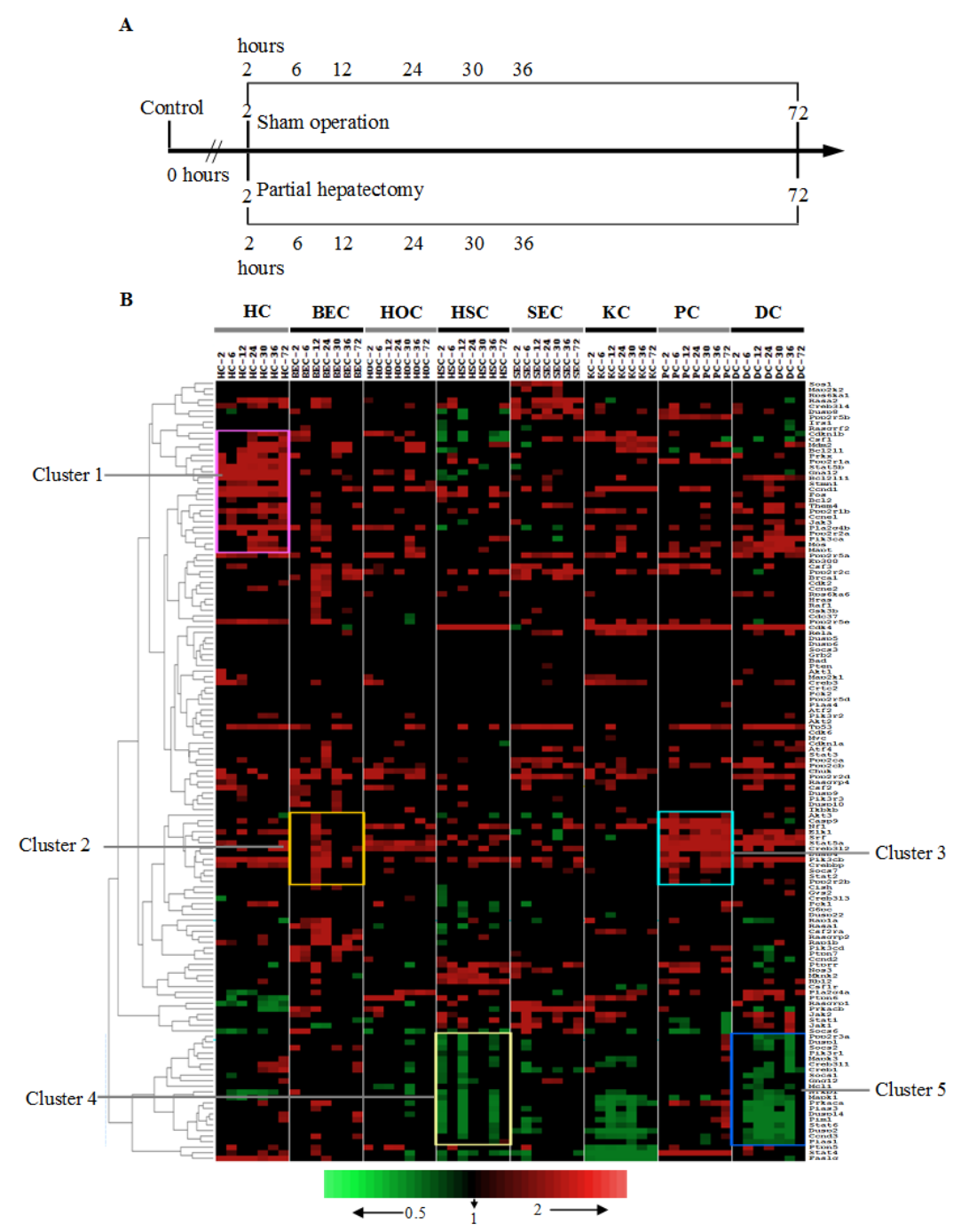

Figure 3. Temporal expression profiling of CSF-mediated signal pathway in 8 liver cells during hepatic regeneration after partial hepatectomy in rats. A. Adult SD rats were shifted to partially surgical resection and subsequently analyzed at indicated times. B. Hierarchical clustering analysis of expression profiles. Each column represents the mean of three replicates for each time sampled. Each row represents one of $141 \mathrm{CSF}-$ mediated signaling pathway-involved genes presented in microarray. The red and green colors represent high, low and insignificant expression relative to control, respectively. Five major clusters, each with an expression correlation, are highlighted in different colored frames. HC $=$ hepatocyte; $\mathrm{BEC}=$ biliary epithelial cell; $\mathrm{HOC}=$ hepatic oval cell; $\mathrm{HSC}=$ hepatic stellate cell; $\mathrm{SEC}=$ sinusoidal endothelial cell; $\mathrm{KC}=$ Kuppfer cell; $\mathrm{PC}=$ pit cell; $\mathrm{DC}=$ dendritic cell. 
To further find out which intercellular cascades are involved in CSF signaling, we manually searched the pertinent signal pathway database and found at least $180 \mathrm{CSF}$-mediated signaling pathway genes. Among the 180 genes, there were 141 genes (which contained 128 differentially expressed genes at least at one time point in any sample) present on the chip. We then performed KEGG-based analysis by using the KEGG software [http://www.genome.jp/kegg/ ma pper.html]. Briefly, we put these 141 genes into the KEGG website to create the KEGG pathway, and the result showed that CSF signals mainly induce the following three intercellular signaling pathways: Ras/Raf/Mapk pathway, Pi3k/Akt pathway and Jak/Stat cascade.

On the basis of the similarity in expression, the 141 CSF-mediated signaling pathway genes across 56 cell samples were clustered as shown in Figure 3B. On the whole, the transcription profiles of these genes differed greatly between the 8 liver cell types after PH. According to the generated heat map, some genes clustered in the confined regions of the heat map. For instance, mRNA levels of some CSF3-induced Pi3k/Akt signaling pathway genes (such as $C S F 3, P I K 3 C A, B C L 2, C C N D 1, C D K 2$, and so on) in hepatocytes were much higher than in other cell types (Cluster 1 in Figure 3B) and gene expression patterns showed significant upregulation during almost the whole LR (Figure 4A). Some CSF2-induced Pi3k/Akt cascade genes including $C S F 2, P I K 3 R 3, A K T 3, I K B K B$, and $C A S P 9$ were mainly upregulated at $12 \mathrm{~h}$ in BEC (Cluster 2 in Figure 3B) and during almost the whole LR in pit cell (PC) (Cluster 3 in Figure $3 \mathrm{~B}$ ), and expression pattern analysis also showed the same expression trends (Figure 4B and C). Based on GO annotation, the above genes are mainly associated with cell proliferation and anti-apoptosis. On the other hand, in contrast to other liver cells, a cluster of genes, mainly including CSF1R, JAK1, STAT6, PTPN6, STAT1, JAK2, SOCS6, etc, were more significantly downregulated at 2, 12, 36 h in hepatic stellate cell (HSC) (Cluster 4 in Figure 3B) and at 6-36 $\mathrm{h}$ in dendritic cells (DC) (Cluster 5 in Figure 3B), which is also shown in Figure $4 \mathrm{D}$ and $\mathrm{E}$. It is known that protein products of these genes are the components of the wellstudied CSF-induced Jak/Stat pathway, which is responsible for cell growth and proliferation.
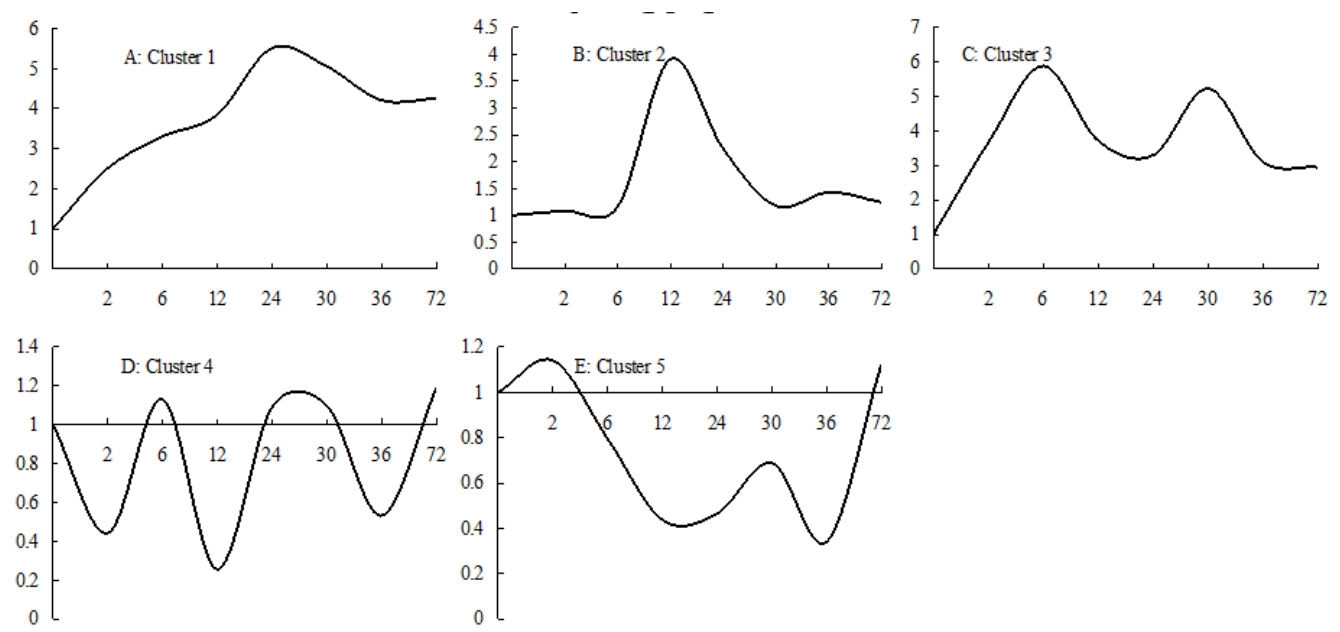

Figure 4. Line graphs showing gene expression patterns of five major clusters. The line graphs are constructed with mean value of mRNA abundance of all genes in each cluster. X-axis represents the recovery times after partial hepatectomy (h); y-axis represents the logarithm of the relative signal values of gene expression. 


\section{CSF-mediated signal transduction activities in $\mathbf{8}$ liver cell types of hepatectomized rat}

To find out whether the expression pattern of the above five CSF-induced signaling pathway genes can reflect the true activity status of these signal pathways in five different liver cells during LR in rats, we used a mathematical model $\left(\mathrm{E}_{\mathrm{t}}\right)$ describing the timing, occurrence and intensity of some biological processes to analyze the synergy of genes participating in CSF-induced signaling pathway in different liver cells during the regenerative process. The results showed that, on the whole, above five CSF-induced signaling pathways were slightly affected in the initial phase, followed by a marked alteration in the proliferative phase. Specifically, the CSF3-induced Pi3k/Akt cascade in hepatocytes had almost no change at 2-6 h after $\mathrm{PH}$, while there was a marked increase in the proliferative phase, especially at 30-36 $\mathrm{h}$ post-PH; in BEC and PC, the CSF2-induced Pi3k/Akt cascade became active only at 12-24 h (belonging to the proliferation phase); CSF1-induced Jak/Stat cascade was sharply reduced at 2-12 $\mathrm{h}$ in HSC, but inactive at in DC during LR (Table 1).

\begin{tabular}{|c|c|c|c|c|c|c|c|c|c|c|c|c|c|c|c|}
\hline \multirow[t]{3}{*}{ CSF-mediated pathway } & \multirow[t]{3}{*}{ Cell types } & \multicolumn{14}{|c|}{ Synergistic effect at each time (h) after partial hepatectomy } \\
\hline & & \multicolumn{2}{|r|}{2} & \multicolumn{2}{|r|}{6} & \multicolumn{2}{|c|}{12} & \multicolumn{2}{|c|}{24} & \multicolumn{2}{|c|}{30} & \multicolumn{2}{|c|}{36} & \multicolumn{2}{|r|}{72} \\
\hline & & $\mathrm{E}_{\mathrm{t}}$ & Result & $E_{t}$ & Result & $\mathrm{E}_{\mathrm{t}}$ & Result & $\mathrm{E}_{\mathrm{t}}$ & Result & $\mathrm{E}_{\mathrm{t}}$ & Result & $\mathrm{E}_{\mathrm{t}}$ & Result & $\mathrm{E}_{\mathrm{t}}$ & Result \\
\hline CSF3-induced Pi3k/Akt signaling pathway & $\mathrm{HC}$ & 10.7 & - & 30.9 & - & 50.1 & - & 29.8 & - & 20.6 & $\uparrow^{*}$ & 43.2 & $\uparrow^{*}$ & 83.9 & - \\
\hline \multirow[t]{2}{*}{ CSF2-induced Pi3k/Akt signaling pathway } & $\mathrm{BEC}$ & 19.3 & - & 34.7 & - & 24.7 & - & 43.0 & $\uparrow^{*}$ & 34.3 & - & 55.0 & - & 64.6 & - \\
\hline & $\mathrm{PC}$ & 77.1 & - & 97.6 & - & 121.9 & $\uparrow^{*}$ & 130.8 & $\uparrow^{*}$ & 110.3 & - & 3.8 & - & -53.6 & - \\
\hline \multirow[t]{2}{*}{ CSF1-induced Jak/Stat signaling pathway } & HSC & -13.9 & $\downarrow^{*}$ & -0.8 & - & 17.7 & $\downarrow^{*}$ & 24.1 & - & 12.9 & - & 4.3 & - & -8.5 & - \\
\hline & DC & 48.3 & - & 43.9 & - & 26.5 & - & 8.2 & - & 6.4 & - & 18.1 & - & 11.5 & - \\
\hline
\end{tabular}

$\mathrm{HC}=$ hepatocyte $\mathrm{BEC}=$ biliary epithelial cell; $\mathrm{PC}=$ pit cell; $\mathrm{HSC}=$ hepatic stellate cell; $\mathrm{DC}=$ dendritic cell. *Significant difference: upward arrow = activity is stronger than control; downward arrow = activity is weaker than control.

\section{DISCUSSION}

Colony stimulating factors are secreted glycoproteins that can bind to receptors on the surfaces of HSCs. Many studies have reported that these growth factors have a positive effect on liver regeneration by inducing intracellular signaling pathways that can cause cell proliferation and differentiation. To better understand how CSF-mediated signaling pathways function in different liver cells after $\mathrm{PH}$, we isolated eight types of high-purity viable liver cells by Percoll density centrifugation and immunomagnetic bead methods (Chen et al., 2010b), and then applied Rat Genome 2302.0 Microarray to measure the expression profiles in eight liver cells at $0,2,6,12,24,30,36$, and $72 \mathrm{~h}$ after PH. Finally, RT-PCR analysis demonstrated the reproducibility of microarray data used in this study.

Amemiya et al. (2011) demonstrated that CSF are secreted mainly by Kupffer cells in the liver. In the literature, there are also reports that, within the regenerating liver, oval cells express CSF3 and its receptor, while sinusoidal endothelial cells (SEC) upregulate expression of CSF2 and its receptor CSF2R (Dame et al., 2002; Meng et al., 2012). A significant production of CSF1 has been discovered in hepatocytes, besides Kupffer cells (Tsukui et al., 1992). However, the present study showed that not only CSF receptors were insignificantly increased or even sharply decreased in mRNA abundance in most of eight liver cells, but also the ex- 
pression of CSF family members in the eight liver cells differed from above findings to some extent. For example, the mRNA abundance of CSF1 was decreased in $\mathrm{HC}$, which was quite opposite to the result of Tsukui et al. (1992); CSF2 was highly expressed during LR in other liver cells except for DC, where this was basically consistent with a previous report; CSF3 was upregulated in other liver cells with the exception of SEC, which is largely consistent with the report by Dame et al. (2002) that both HC and nonparenchymal cells can secrete CSF. Notably here, there is very little evidence that CSF are synthesized in BEC, but it is interesting that our results showed a high expression of CSF1-3 in BEC.

As previously stated, the postoperative administration of CSF 1,2 or 3 promotes the proliferation of hepatic cells by inducing intracellular cascades, thus contributing to liver regeneration (Kaushansky, 2006; Akagawa et al., 2006; Piscaglia et al., 2007). To clarify how CSF impact the regeneration process at the cellular level, we performed high throughput analysis to measure expression profiles of CSF-mediated signaling pathway genes in eight hepatic cell types following PH. The results showed that different cell types greatly differed in gene transcriptional profiles. Specifically, a major proportion of genes involved in CSF3-mediated signaling pathways (especially Pi3k/Akt pathway) in $\mathrm{HC}$ were markedly upregulated during almost the whole LR (Figure 4). Likewise, most of the genes involved in CSF2-mediated signaling pathways (especially Pi3k/Akt pathway) in BEC were increased at the mRNA level mainly at $12 \mathrm{~h}$ post-PH, and also in PCs during almost the whole LR. On the other hand, HSC and DC showed significant downregulation of the genes associated with the CSF1-induced $\mathrm{Jak} /$ Stat cascade 2-36 h following PH, which could be explained by the fact that the promotion of cell proliferation is via signal pathways other than the CSF2-induced Jak/Stat pathway. Here, it needs to be emphasized that Kupffer cells, the main CSF-secreting cells, showed marked downregulation of a group of genes involved in the CSF1-inducing Jak/Stat pathway during almost the whole LR, which needs a reasonable experimental explanation.

Anyway, it is almost impossible to systemically define the timing, occurrence and strength of the above five signaling pathways in different liver cells during LR only by examining expression profiles of the related genes. To assess their combining effect, the abovementioned mathematical model measuring gene synergy was used to test the signal transduction activities induced by CSF in the above liver cells during LR in rats. The results of gene synergy showed that the CSF3-induced Pi3k/Akt pathway in HC was significantly enhanced at 30 and $36 \mathrm{~h}$ post-PH. Similarly, the CSF2-induced Pi3k/Akt pathway in BEC was increased mainly at $24 \mathrm{~h}$ post-PH, and this pathway was activated at 12-24 h in PC. In HSC, it turned out that the Jak/Stat cascade was not active or even strikingly decreased at 2 and $12 \mathrm{~h}$ post-PH, and not significantly different compared to the control in DC. What needs to be mentioned is that in Kupffer cells, CSF-mediated signaling pathways including Ras/Raf/Mapk, Pi3k/Akt and $\mathrm{Jak} / \mathrm{Stat}$ became inactive during the whole LR. According to previous studies, CSF could improve liver repair and restoration, implicating the relative active signal pathway triggered by them (Piscaglia et al., 2007). However, the difference between our results and those of other studies suggests the possibility that the inactive CSF-mediated signaling pathways during LR may be try to prevent the regenerating liver from inflammatory injury caused by CSF, or this difference is related to the difference between tissue-level studies and cell-level studies. Since the data available are limited, the reasons require further experimental investigation.

Briefly, we performed a large-scale analysis of gene expression profiles, and obtained some detailed data on rat liver regeneration at the cellular level. These data suggest the po- 
tential relevance between CSF and LR at the cellular level. In addition, according to gene synergy, the number of differentially expressed genes partly but not completely reflected the strength of signaling pathway, reinforcing the viewpoint that some biological activity is the outcome of the synergy of a group of functionally related genes. However, the above results are mainly drawn from gene expression profiles measured by GeneChip. Since a microarray can only test for gene transcription, it cannot reflect the protein synthesis process, even less able to accurately reflect CSF-mediated signaling pathway activities and the final biological effect. Therefore, in the future, we need to verify the above results using methods such as protein interaction, gene addition, RNA interference, etc.

\section{ACKNOWLEDGMENTS}

Research supported by the National Basic Research "973" Pre-Research Program of China (\#2010CB534905) and the Doctoral Startup Foundation of Henan University of Science and Technology (\#09001579).

\section{REFERENCES}

Akagawa KS, Komuro I, Kanazawa H, Yamazaki T, et al. (2006). Functional heterogeneity of colony-stimulating factorinduced human monocyte-derived macrophages. Respirology (Suppl 11): S32-S36.

Amemiya H, Kono H and Fujii H (2011). Liver regeneration is impaired in macrophage colony stimulating factor deficient mice after partial hepatectomy: the role of M-CSF-induced macrophages. J. Surg. Res. 165: 59-67.

Bhanu NV, Trice TA, Lee YT and Miller JL (2004). A signaling mechanism for growth-related expression of fetal hemoglobin. Blood 103: 1929-1933.

Chen X and Xu C (2012). High-throughput analysis of tumor necrosis factor signaling pathways in eight cell types during rat hepatic regeneration. Inflammation 35: 1538-1548.

Chen X, Xu C, Zhang F and Ma J (2010a). Microarray approach reveals the relevance of interferon signaling pathways with rat liver restoration post 2/3 hepatectomy at cellular level. J. Interferon Cytokine Res. 30: 525-539.

Chen X, Xu C, Zhang F and Ma J (2010b). Comparative analysis of expression profiles of chemokines, chemokine receptors, and components of signaling pathways mediated by chemokines in eight cell types during rat liver regeneration. Genome 53: 608-618.

Christ B and Pelz S (2013). Implication of hepatic stem cells in functional liver repopulation. Cytometry A 83: 90-102.

Dame JB, Chegini N, Christensen RD and Juul SE (2002). The effect of interleukin-1beta (IL-1beta) and tumor necrosis factor-alpha (TNF-alpha) on granulocyte macrophage-colony stimulating factor (GM-CSF) production by neuronal precursor cells. Eur. Cytokine Netw. 13: 128-133.

Gaia S, Smedile A, Omede P, Olivero A, et al. (2006). Feasibility and safety of G-CSF administration to induce bone marrow-derived cells mobilization in patients with end stage liver disease. J. Hepatol. 45: 13-19.

Higgins GM and Anderson RM (1931). Experimental pathology of the liver I: restoration of the liver of the white rat following partial surgical removal. Arch. Pathol. 12: 186-202.

Ishikawa T, Aoshiba K, Yokohori N and Nagai A (2006). Macrophage colony-stimulating factor aggravates rather than regenerates emphysematous lungs in mice. Respiration 73: 538-545.

Kang W, Liu ZW, Han QY, Zhang L, et al. (2008). Effects of granulocyte colony-stimulating factor on hepatocyte apoptosis in acute liver failure: experiment with rats. Zhonghua Yi Xue Za Zhi 88: 980-984.

Kaushansky K (2006). Hematopoietic growth factors, signaling and the chronic myeloproliferative disorders. Cytokine Growth Factor Rev. 17: 423-430.

Lin XM, Liu YB, Zhou F, Wu YL, et al. (2008). Expression of tumor necrosis factor-alpha converting enzyme in liver regeneration after partial hepatectomy. World J. Gastroenterol. 14: 1353-1357.

Meng F, Francis H, Glaser S, Han Y, et al. (2012). Role of stem cell factor and granulocyte colony-stimulating factor in remodeling during liver regeneration. Hepatology 55: 209-221.

Nault R, Kim S and Zacharewski TR (2013). Comparison of TCDD-elicited genome-wide hepatic gene expression in Sprague-Dawley rats and C57BL/6 mice. Toxicol. Appl. Pharmacol. 267: 184-191. 
Ogiso T, Nagaki M, Takai S, Tsukada Y, et al. (2007). Granulocyte colony-stimulating factor impairs liver regeneration in mice through the up-regulation of interleukin-1beta. J. Hepatol. 47: 816-825.

Ono M, Yu B, Hardison EG, Mastrangelo MA, et al. (2004). Increased susceptibility to liver injury after hemorrhagic shock in rats chronically fed ethanol: role of nuclear factor-kappa B, interleukin-6, and granulocyte colony-stimulating factor. Shock 21: 519-525.

Piscaglia AC, Shupe TD, Oh SH, Gasbarrini A, et al. (2007). Granulocyte-colony stimulating factor promotes liver repair and induces oval cell migration and proliferation in rats. Gastroenterology 133: 619-631.

Ruse M and Knaus UG (2006). New players in TLR-mediated innate immunity: PI3K and small Rho GTPases. Immunol. Res. 34: 33-48.

Sidler D, Studer P, Kupper S, Gloor B, et al. (2008). Granulocyte colony-stimulating factor increases hepatic sinusoidal perfusion during liver regeneration in mice. J. Invest. Surg. 21: 57-64.

Tsukui T, Kikuchi K, Mabuchi A, Sudo T, et al. (1992). Production of macrophage colony-stimulating factor by adult murine parenchymal liver cells (hepatocytes). J. Leukoc. Biol. 52: 383-389.

Van Sweringen HL, Sakai N, Tevar AD, Burns JM, et al. (2011). CXC chemokine signaling in the liver: impact on repair and regeneration. Hepatology 54: 1445-1453.

Wang GP and Xu CS (2010). Reference gene selection for real-time RT-PCR in eight kinds of rat regenerating hepatic cells. Mol. Biotechnol. 46: 49-57.

Wang WB, Fan JM, Zhang XL, Xu J, et al. (2009). Serial expression analysis of liver regeneration-related genes in rat regenerating liver. Mol. Biotechnol. 43: 221-231. 